\title{
Corela
}

Cognition, représentation, langage

HS-28 | 2019

Les affordances langagières : textualité numérique, matérialité discursive

\section{Technographismes en ligne. Énonciation matérielle visuelle et iconisation du texte}

\section{Marie-Anne Paveau}

\section{CpenEdition}

Journals

Édition électronique

URL : http://journals.openedition.org/corela/9185

DOI : $10.4000 /$ corela.9185

ISSN : 1638-573X

Éditeur

Cercle linguistique du Centre et de I'Ouest - CerLICO

Référence électronique

Marie-Anne Paveau, «Technographismes en ligne. Énonciation matérielle visuelle et iconisation du texte », Corela [En ligne], HS-28 | 2019, mis en ligne le 11 septembre 2019, consulté le 13 septembre 2019. URL : http://journals.openedition.org/corela/9185; DOI : 10.4000/corela.9185

Ce document a été généré automatiquement le 13 septembre 2019

\section{cc) (†)(ด)}

Corela - cognition, représentation, langage est mis à disposition selon les termes de la licence Creative Commons Attribution - Pas d'Utilisation Commerciale - Partage dans les Mêmes Conditions 4.0 International. 


\title{
Technographismes en ligne. Énonciation matérielle visuelle et iconisation du texte
}

\author{
Marie-Anne Paveau
}

\section{Introduction}

1 J'écris ces lignes le 4 août 2018, jour anniversaire de l'accession au pouvoir de Thomas Sankara. Le web social étant, entre autres, un web commémoratif, je trouve immédiatement en consultant mon fil Twitter des composés image-textes de ce type : 


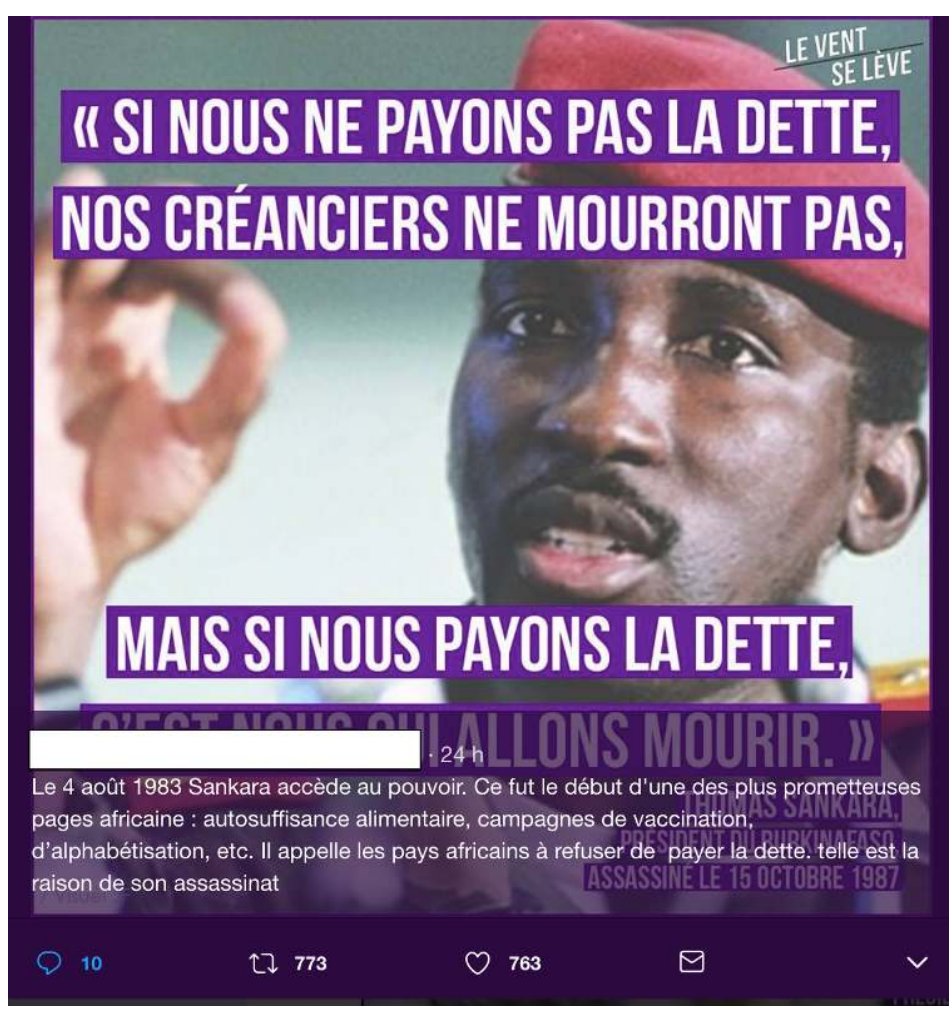

Image 1 : Image incrustée diffusée le 04.08.2018, fil Twitter M.-A. Paveau

2 C'est ce type de composition que j'appelle technographisme. La conversion numérique de nos activités discursives, en particulier sur le web social, a pour conséquence une iconisation de la communication, c'est-à-dire une utilisation de l'image qui dépasse la simple illustration pour devenir une véritable porteuse de sens. Le technographisme, qui est un composite image-texte natif du web, en est l'une des meilleures preuves. C'est à cette composition numérique native au fonctionnement particulier qu'est consacré cet article.

3 Mon travail se situe dans la cadre de l'analyse du discours numérique que j'ai mise en place pour rendre compte des discours numériques natifs, c'est-à-dire produits dans les écosystèmes d'internet, en particulier du web dit 2.0 (Paveau 2017a) ${ }^{1}$. Mon approche repose sur la notion de technologie discursive, définie comme un dispositif au sein duquel la production discursive est intrinsèquement liée à des outils et gestes technologiques qui participent à la mise en forme des matières langagières constitutives du discours, dans des environnements connectés (Paveau 2013). Le cadre épistémologique est celui du postdualisme (remise en cause de la distinction langue/monde et des ordres linguistique/ extralinguistique), qui motive une linguistique symétrique (pour laquelle les environnements de production des discours ne sont pas distincts d'eux mais dans un rapport de continuum égalitaire ${ }^{2}$ ). Les environnements dans leurs ensemble constituant l'objet de l'investigation, et non plus les seuls discours extraits de leurs milieux de vie, l'analyse du discours numérique se définit comme une écologie du discours qui veut rendre compte de la conversion numérique de l'ensemble des activités discursives (Doueihi 2008). Les discours sont produits dans les environnements connectés, dans les machines et les outils logiciels d'écriture, ces derniers n'étant pas considérés comme de simples médiations, ce qui reconduirait l'autonomie des discours, autrement dit une forme de logocentrisme ${ }^{3}$. L'analyse du discours numérique a donc pour but de 
prendre acte des conversions comme des modifications des activités discursives appelant des aménagements épistémologiques et théoriques.

4 Après une présentation du technographisme, je définirai les notions d'énonciation visuelle et d'iconisation du texte, puis je prendrai l'exemple de la photographie déictique de texte pour montrer comment, dans les productions numériques natives, l'ordre textuel et l'ordre iconique s'articulent de manière composite.

\section{Le technographisme, un composite numérique natif}

5 Je propose six principes de fonctionnement pour caractériser les discours numériques natifs : la composition, la délinéarisation, l'augmentation, la relationalité, l'investigabilité et l'imprévisibilité (Paveau 2017a). Pour aborder le technographisme, je vais surtout mobiliser le principe de composition qui rend compte de la nature structurellement hétérogène des éléments technolangagiers.

\subsection{Technographisme, composition et affordances du web}

Un élément de discours composite est constitué d'un assemblage entre du langagier et du technologique d'ordre bureautique (la machine) et logiciel (le programme, le code, les algorithmes) (Paveau 2015a). J'emprunte le terme assemblage à l'anthropologie symétrique de Bruno Latour qui décrit ainsi l'articulation entre l'ordre social et l'ordre naturel qui caractérise tout hybride (Latour 1991). Le terme composite décrit le résultat da coconstitution du langagier et du technologique dans les discours numériques natifs. Cette notion est construite à partir de trois références majeures qui fondent l'approche postdualiste : l'externalité de l'esprit qui postule que nous pensons à partir d'éléments extérieurs à notre esprit (Clark \& Chalmers 1998), la cognition distribuée qui pose que la construction de représentations et la transmission d'informations se fait à travers des outils et des objets extérieurs à la conscience humaine (Hutchins 1995) et la théorie des affordances qui propose de penser les objets comme des producteurs intentionnels d'indications sémantiques (Gibson 1977, Norman 1993, Dervin et Paveau 2012 dir., Paveau 2012).

7 Une affordance est l'ensemble des possibilités d'usage offertes par un objet; elle repose sur la perception humaine, induit une adaptation à l'environnement et postule une forme d'agentivité des objets. La théorie des affordances suppose une épistémè non dualiste et une conception non symbolique de l'information (c'est-à-dire ouverte vers l'icône et l'indice, selon les catégories peirciennes). La notion d'affordance a été mise en jeu dans différents travaux pour rendre compte du rôle des dimensions objectales et/ou techniques du contexte, notamment en cognition distribuée, en sociologie cognitive, dans l'interactionnisme (en particulier les travaux du groupe IMPEC) et en analyse du discours. Elle connaît une extension de sens dans les études du numérique, définissant les possibilités techniques des écosystèmes de l'internet ou plus spécifiquement du web (programmes, CMS, outils, plateformes, etc.), c'est-à-dire ce qui est mis à disposition des usager.e.s sur le plan technologique pour produire des contenus en ligne. Les affordances impliquent des liens aux appareils, la présence de l'énonciateur.trice comme du.de la récepteur.trice étant nécessaire à l'existence matérielle du texte produit via le fonctionnement appareil: c'est ce qui différencie fondamentalement le texte prénumérique hors ligne du texte numérique en ligne comme le souligne Emmanuël 
Souchier dès les années 1990 (Souchier 1996). Pour résumer, les contenus en ligne, et particulièrement les technographismes envisagés ici, sont des produits affordanciels, les liens matériels qu'ils entretiennent avec les outils du web ayant effets discursifs et scripturaux. Par conséquent, les observables de recherche ne sont plus seulement les matières langagières, dans une perspective logocentrée, mais des matières composites, coconstituées de non-langagier de nature technique, regardées dans une perspective écologique (Paveau 2017b).

\subsection{Définition du technographisme}

Parmi ces productions composites, le technographisme, composite de texte et d'image fixe ou animée tient une place importante dans les productions discursives natives du web, constituant d'ailleurs une partie de sa culture. Il s'agit par exemple d'images macro (image 1), d'images incrustées de textes, devenues courantes dans la communication politique (image 2), de pancartes numériques (image 3), de tickers, signatures d'internautes sur les forums (image 4). Ces productions, hormis peut-être les images macro pace qu'elles sont des types de mèmes, ne sont pas encore analysées en sciences du langage et très peu en sciences humaines et sociales en général, et donc peu identifiées ; l'étiquette pancarte numérique m'est personnelle par exemple.

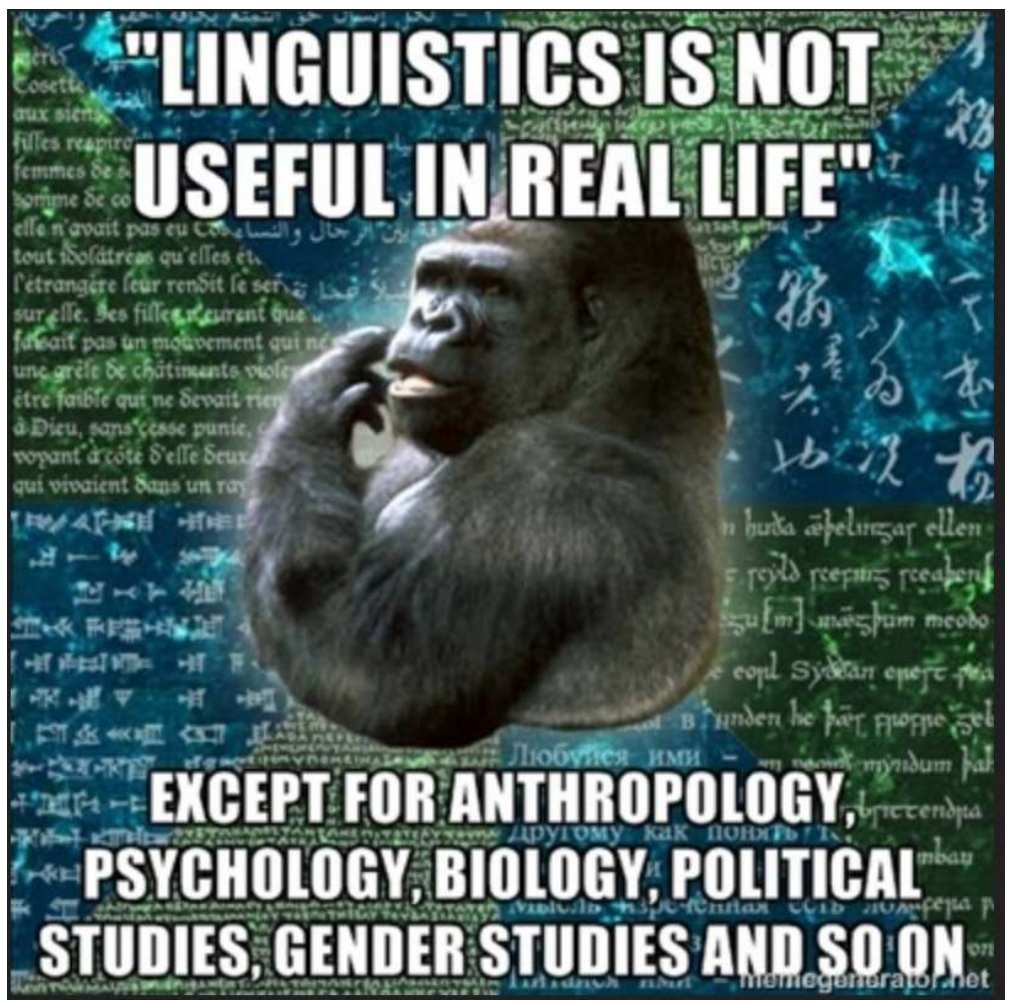

Image 2 : image-macro (source : https://78.media.tumblr.com) 


\section{La France a les moyens} de devenir de nouveau une puissance du XX|e siècle.

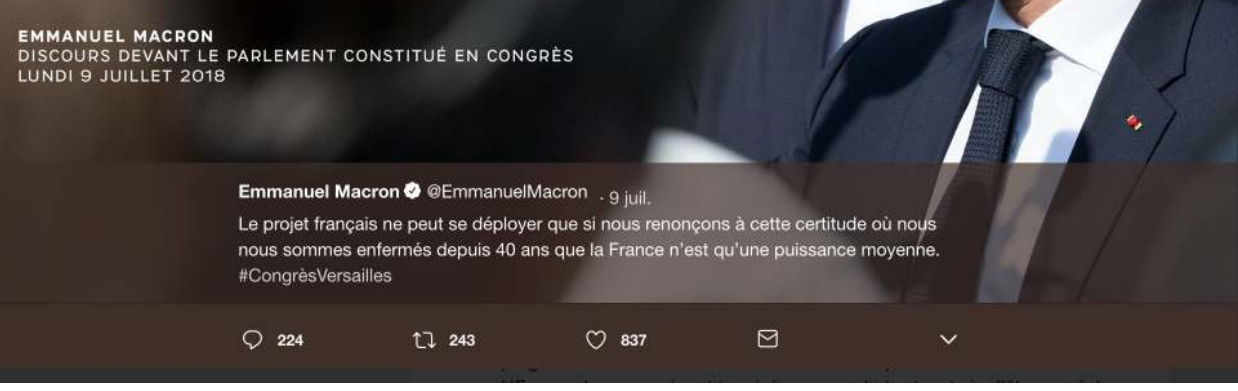

Image 3 : image incrustée de texte (source : compte Twitter du Président de la république, 09.07.18)

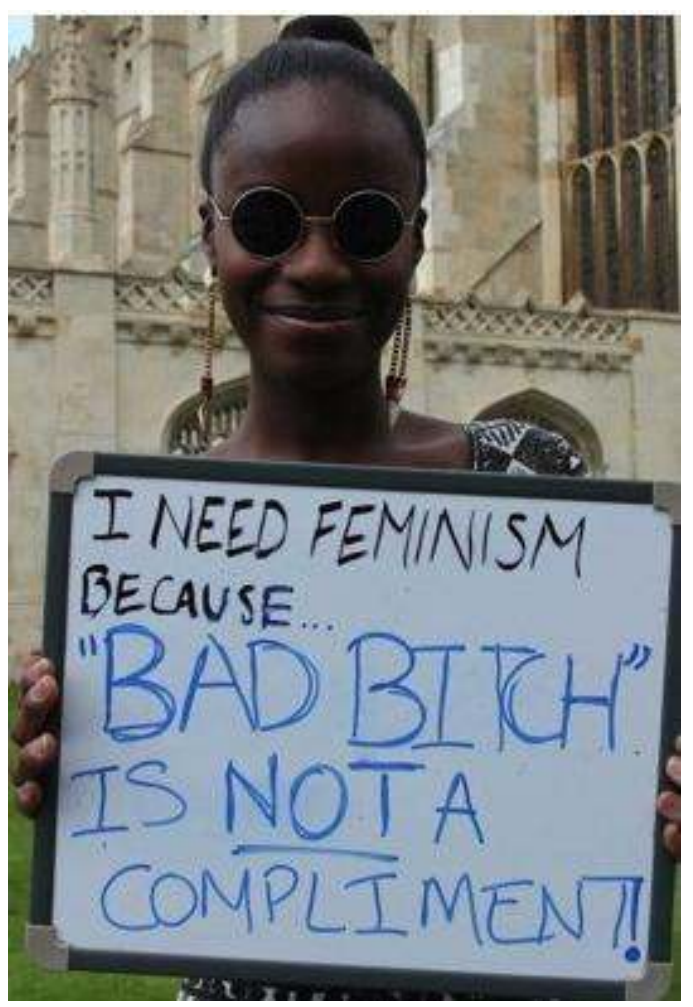

Image 4 : pancarte numérique issue de la campagne \#IneedFeminism à Cambridge en juin $2013^{4}$ 


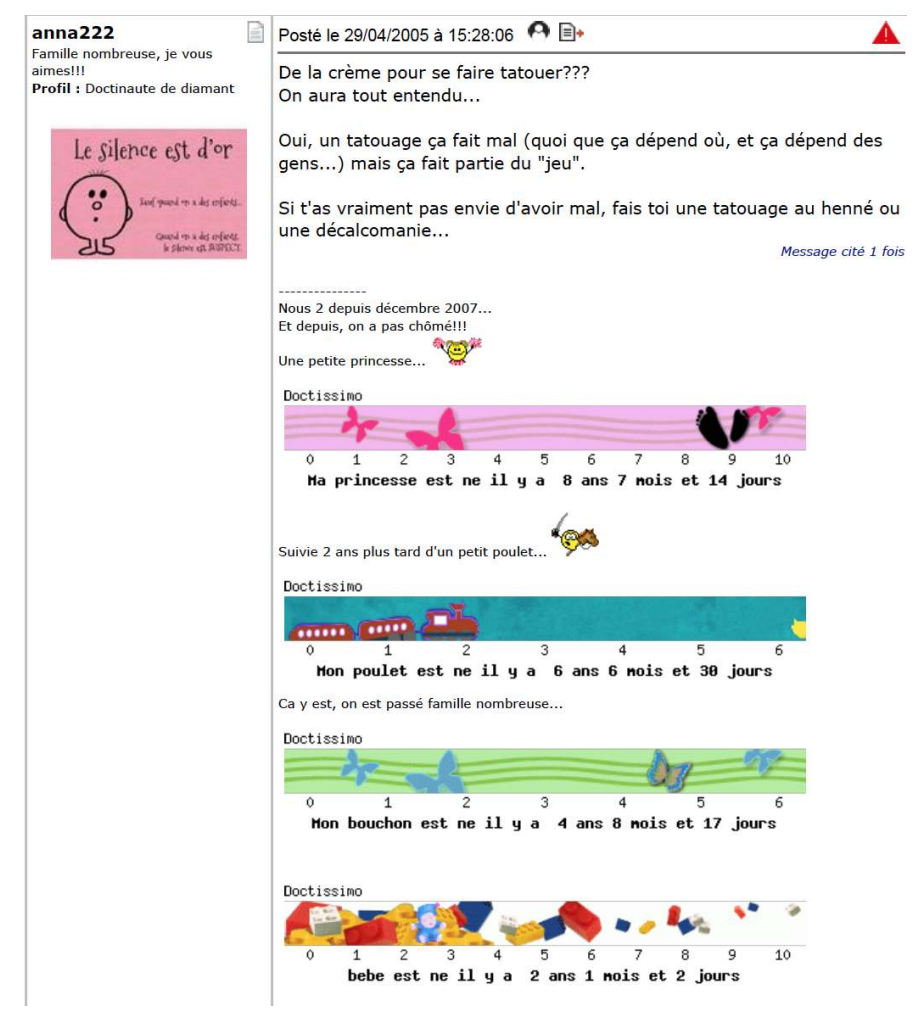

Image 5 : ticker d'une forumeuse sur le forum « Tatouage » de Doctissimo

9 Je définis plus précisément le technographisme comme une production plurisémiotique associant texte et image dans un composite multimédiatique natif d'internet, produit par des outils et des gestes technologiques et entré dans les normes des discours numériques natifs. Multimédiatique signifie issu d'une «manipulation conjointe de textes, images et sons sur un même support» (Bouchardon et al. 2011: 12). Et je choisis l'élément graphisme pour englober le geste de tracer (dessin ou image) et le geste d'écrire (texte). Le technographisme est en fait un double composite : il est technodiscursif (technologie et langage) et verbo-iconique (langage et image). Il manifeste la dimension multimédiatique de la littéracie en ligne (c'est-à-dire les systèmes d'écriture et les productions sémiotiques) en présentant un assemblage de différents codes dans des réalisations intrinsèquement et simultanément plurisémiotiques.

10 L'observation des composés image-texte n'est pas nouvelle et en 1990 Michael Nerlich avait proposé la notion d'iconotexte, définie à partir de la combinaison sous forme d'une coprésence du texte et de l'image, caractéristique de la bande dessinée, de l'album ou du cinéma). Michael Nerlich travaille dans l'ordre du livre et décrit l'iconotexte comme « une unité indissoluble de texte(s) et image(s) dans laquelle ni le texte ni l'image n'ont de fonction illustrative et qui - normalement, mais non nécessairement - a la forme d'un "livre" » (Nerlich 1990). Le technographisme ne peut répondre à cette définition pour trois raisons : d'abord, internet et le web n'appartiennent pas à l'ordre du livre mais surtout, l'assemblage image et texte n'y est pas une coprésence, ce qui impliquerait l'autonomie des deux éléments, mais une véritable co-intégration, dans laquelle aucun des éléments n'est extractible ou observable séparément des autres; enfin, la dimension technologique logicielle du technographisme est absente de l'iconotexte.

11 Je pose deux questions à propos du technographisme. Une question générale, celle de la production de sens, dont j'emprunte la formulation à Serge Bouchardon et al. : « comment 
des médias différents (texte, image, son, vidéo) vont-ils faire signe et sens ensemble dans un contexte de communication donné ?» (Bouchardon et al. 2011:16). Et une question plus spécifique : que fait l'image au texte? que deviennent, comment se comportent les discours numériques pris dans une énonciation visuelle par iconisation du texte?

\section{Une énonciation matérielle visuelle}

\subsection{De l'énonciation éditoriale à l'énonciation visuelle : le pictorial turn}

On doit la notion d'énonciation éditoriale à Emmanuël Souchier qui, au début des années 1990, se saisit de la question des écritures électroniques et de la composition des pages internet dans l'article fondateur où il élabore la notion non moins fondatrice d'écrit d'écran. Il y précise qu'«il s'agit de ne pas considérer le texte en dehors de sa réalité matérielle et sociale et de ne pas envisager l'œuvre "en soi", mais en situation (selon ses conditions de production, de diffusion ou de réception) "(Souchier, 1998: 1). L'énonciation éditoriale, définie plus tard avec Yves Jeanneret, intègre à la production proprement textuelle les conditions matérielles de son élaboration: "L'énonciation éditoriale désigne l'ensemble de ce qui contribue à la production matérielle des formes qui donnent au texte sa consistance, son "image de texte". Il s'agit d'un processus social déterminé, qui demeure largement invisible du public, mais qui peut néanmoins être appréhendé à travers la marque qu'impriment les pratiques de métiers constitutives de l'élaboration, de la constitution ou de la circulation des textes. [...] Plus fondamentalement, l'énonciation éditoriale est ce par quoi le texte peut exister matériellement, socialement, culturellement... aux yeux du lecteur » (Jeanneret, Souchier $2005: 6)$.

13 L'usage massif du technographisme en ligne et l'importance croissante de l'image dans tous les espaces de communication écrite du web, notamment les réseaux sociaux et les applications connectées (du type Whatsapp), me semble motiver l'idée d'une énonciation éditoriale où l'image aurait une place prépondérante: une énonciation visuelle. Le « tournant iconique » (pictorial turn) de l'écriture et de la littérature, tel qu'il est proposé par William J. T. Mitchell dès les années 1990 appuie cette proposition (Mitchell 2009 [1986], 1994). Cette hypothèse constitue une contribution importante du champ des visual studies à l'analyse du discours numérique: la culture visuelle y est vue comme une approche perceptive, l'image organisant notre perception sémiotique, voire dominant le langage articulé, comme l'explique Magali Nachtergael :

Le pictorial turn n'exclut pas pour autant une part linguistique et narrative, bien au contraire, il les articule simplement selon une autre perspective, prioritairement visuelle. Ainsi on peut mettre sur le compte de cette puissance de l'image l'apparition d'études intermédiales et transmédiales qui mettent en avant l'interchangeabilité des supports (livre, écrans, performances, expositions) mais aussi une interprétation du monde guidée non plus par les oppositions syntagmatiques et paradigmatiques, ni sur la structure narrative mais plutôt sur une appréhension englobante et directe telle que la structure en « image » l'impose (Nachtergael 2017 : 293).

14 Autrement dit, en termes linguistiques, c'est l'image qui pilote le sens prioritairement et non plus les formes langagières: avec l'hypothèse du tournant iconique on sort $d u$ 
logocentrisme dans lequel la linguistique reste trop souvent enfermée, ce qui l'empêche sans doute de prendre la pleine mesure des questions soulevées par les discours numériques natifs. Intégrer le pictorial turn à la réflexion linguistique, c'est poser une production de sens de l'image dans le cadre d'une énonciation matérielle visuelle

La réflexion sur la dimension discursive de l'image en ligne est présente et théorisée en études visuelles en France, notamment dans les travaux de Laurence Allard et d'André Gunthert, ce dernier proposant la notion de conversationnalisation de l'image. Dans un mouvement symétrique, les textes deviennent images en ligne, ce qui m'amène à proposer l'idée d'une iconisation du texte. Ce sont ces deux « devenirs » de l'image et du texte que je synthétise dans la notion d'énonciation matérielle visuelle.

\subsection{La conversationnalisation de l'image}

André Gunthert définit l'image conversationnelle comme une photographie « devenue un composant parmi d'autres de l'univers de la communication", dont la caractéristique principale est d'être « embarquée dans chaque objet connecté » (Gunthert, 2014 : § 20). En traduisant ses propos en linguistique, on pourrait dire que l'image est une unité distinctive de conversation, à l'instar d'un élément discursif. Et effectivement, il existe un usage massif de l'image conversationnelle sur internet, dont certaines son devenues des stéréotypes de la publication sur les réseaux, comme les selfies (Allard 2014a, Gunthert 2015), les photos de pieds ou de nourriture. D'autres sont plus contingentes comme les photos de neige analysées par André Gunthert dans un texte intitulé "Il neige sur Facebook », dans lequel il montre que la neige tombant sur la région parisienne, par son caractère exceptionnel, constitue un "événement conversationnel à part entière " (Gunthert 2013 : en ligne). Selon lui, un processus de conversationnalisation de l'image a donc été accompli depuis une dizaine d'années par le web social qui a produit «l'image conversationnelle, produit inattendu de la rencontre de la numérisation des contenus visuels et de l'interaction documentée " $(2014$, § 4). Cette conversationnalisation est permise par des facilités technologiques et elle a donc une dimension affordancielle, puisque «la numérisation, en réduisant la matérialité des images, leur confère une plasticité et une mobilité nouvelles » (2014: § 6).

L'image conversationnelle est désormais redocumentée ${ }^{5}$ et le compte Instagram @Instarepeat propose des séries de photos conversationnelles analogues, comme le montre cette collection de photos de pieds : 


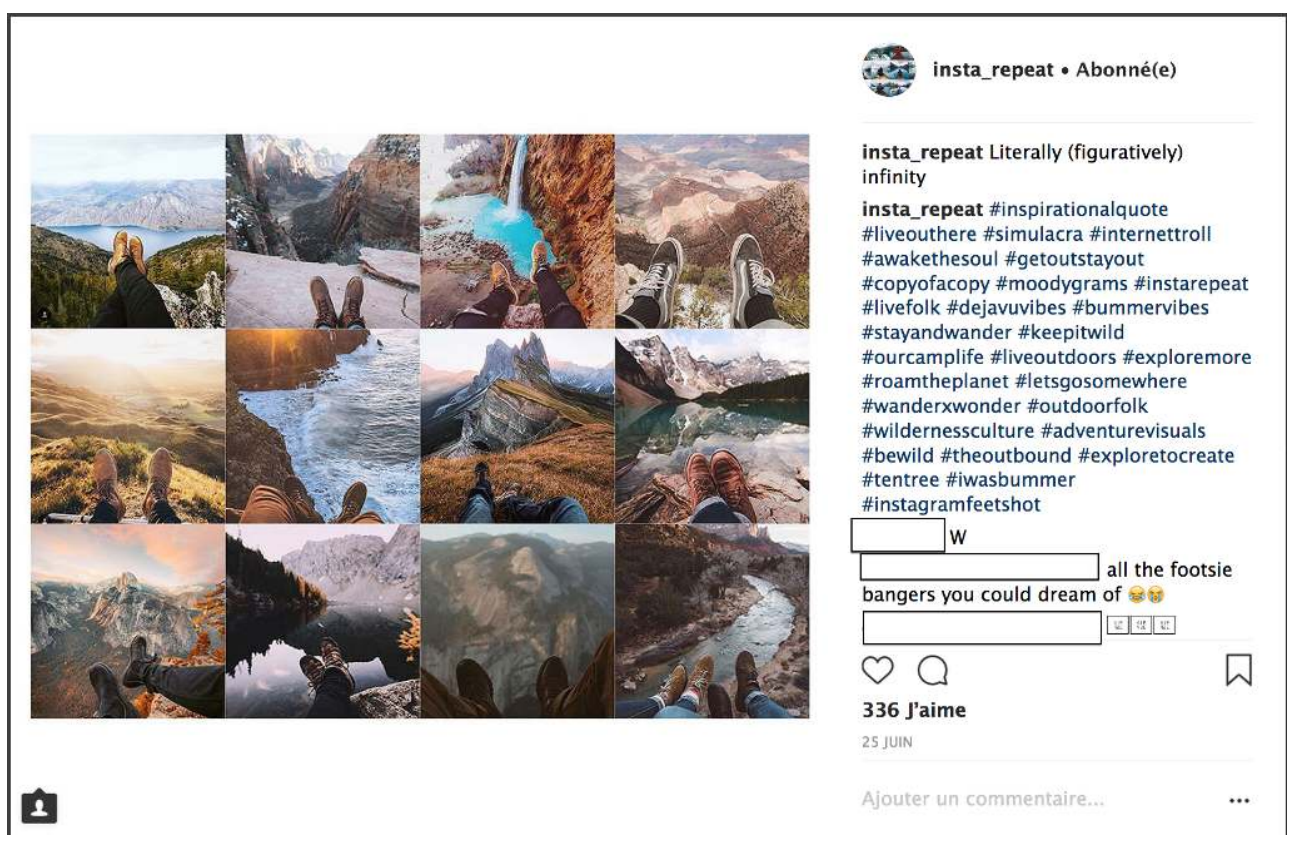

Image 6. Collection sur le compte Instagram @instarepeat, 25.06.18

18 Avec l'image conversationnelle, on reste cependant dans l'ordre iconique seul, le " message » verbal étant de l'ordre de l'interprétation contextuelle à partir de signes non langagiers. Céline Cabourg et Boris Manenti décrivent par exemple les pratiques adolescentes de photos informationnelles : «Se raconter, ils le font en textes, mais aussi évidemment en images. Ils multiplient ainsi les instantanés sans intérêt esthétique, reflets de moments anecdotiques : la bouteille de coca et les boîtes de pizza pour dire qu'on est en train de déjeuner (en ajoutant un bandeau avec des émojis), par exemple. [... ], D'autres sont plus informatives, comme ce gros plan sur les pieds d'un jeune chaussé de baskets qui marchent en forêt, pour signifier une balade (Cabourg, Manenti 2017 : 47). Je retiens donc la notion de conversationnalisation et l'idée que l'image produit du sens, mais je m'intéresse plutôt à des compositions qui tendent en sens inverse à donner à la textualité des caractéristiques iconiques.

\subsection{L'iconisation du texte : faire texte avec l'image}

19 Les technographismes sont devenues des formes ordinaires des discursivités numériques du web social, comme le montrent les travaux d'André Gunthert et Laurence Allard, appuyés sur des enquêtes ethnographiques. Ma propre observation des trois grands réseaux Twitter, Facebook et Instagram tient plus de " l'observation flottante ${ }^{6}$ que de l'enquête proprement dite mais il suffit à mon objectif de description morphologique et énonciative du technographisme, qui n'a pas de prétention généralisante. Je travaille donc à partir d'exemples et non d'un corpus visant une systématisation des résultats. Mon périmètre d'observation est plutôt lettré puisque je travaille à partir de mes propres comptes en grande partie déterminés comme tous les comptes par une certaine homophilie des réseaux ${ }^{7}$ : je vais donc donner des exemples d'usages d'universitaires, d'intellectuels, de journalistes, de militant.e.s, etc., plutôt détenteur.trice.s d'une littéracie forte, caractérisée par une expertise dans les pratiques d'écriture, de mise en forme et de contextualisation culturelle de leurs publications. 
20 À partir de ces exemples, j'interroge les évolutions actuelles du texte en prenant en compte les récentes évolutions de sa définition : en suivant Jean-Michel Adam, je définis le texte non plus prioritairement et exclusivement par ses composantes intrinsèques mais par le processus d'élaboration accompli par le.a lecteur.trice, c'est-à-dire comme un «faire texte» (Adam dir. 2015, Paveau 2015b). Dans cette perspective, le texte résulte de la textualisation du.de la lecteur.trice et des formes non canoniques comme le graffiti, la liste ou le brouillon, et, dans les univers numériques natifs, des formes comme les commentaires, les tweets ou les suites de hashtags (comme dans l'image 6) et évidemment les technographismes, peuvent faire texte si elles sont textualisées par l'écrilecture ${ }^{8}$ de l'internaute. Dans le processus du faire texte contemporain au sein des univers numériques natifs, il me semble que l'on doit faire entrer une nouvelle dimension, qui est l'image : le technographisme montre que le texte est de plus en plus rarement produit hors de l'image qui, loin de l'accompagner comme une illustration ou de l'auxiliarisation en lui donnant statut de légende, l'iconise littéralement. Je définis donc l'iconisation du texte en ligne comme l'élaboration et la mise en forme d'un énoncé dans le format et le code représentationnel de l'image, au moyen d'outils affordanciels dédiés; dans le texte iconisé, les codes textuel et iconique sont coconstitués en composite et ne font pas l'objet d'une lecture ou d'une analyse indépendante.

21 Il faut ajouter que le faire texte ainsi envisagé ne laisse pas les seul.e.s producteur.trice.s et lecteur.trice.s en charge de la textualisation, comme le suppose la linguistique textuelle traditionnelle. Les outils affordanciels du web, désormais largement disponibles et aisément utilisables par chacun.e, sont coresponsables de la production de textes iconisés. On trouve en effet en abondance sur le web des générateurs de mèmes, d'images macro, de vignettes, des outils d'incrustation de texte sur images, des générateurs de photos de profils, d'avatars, de bannières, de filtres (identité numérique) et des 
fonctionnalités permettant d'iconiser les éléments langagiers, comme la fonction d'iconisation des posts de Facebook :

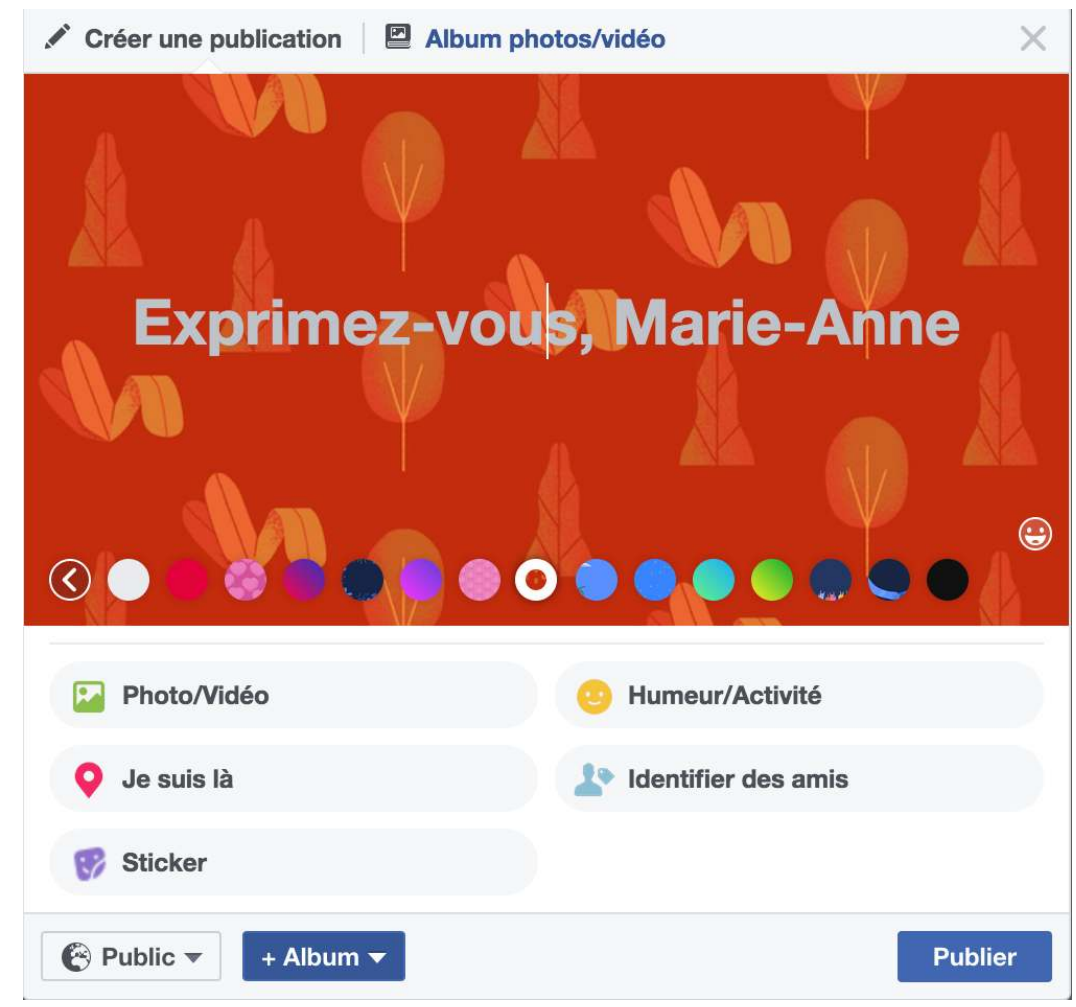

Image 7. Iconisation des posts sur Facebook

$\mathrm{Au}$ lieu d'écrire un post classique dans la fenêtre prévue, je peux en effet choisir une couleur et un motif de fond qui me permet d'élaborer une image dans lequel mon texte est iconiquement mis en forme et incrusté.

L'ensemble de ces possibilités et de ces réalisations produit une acculturation du.de la scripteur.e qui prend acte des modifications des formes de texte et, au bout du compte, de la conversion visuelle du texte. Pour illustrer ce phénomène, je choisis d'examiner un exemple particulier qui relève autant de l'image conversationnelle que de l'iconisation du texte, la photographie déictique de texte.

\section{La photographie déictique de texte}

J'envisage ici l'iconisation du texte par sa photographie et sa mise en circulation dans l'écosystème du web social. Photographier un texte et le diffuser aussitôt est devenu une pratique habituelle grâce aux smartphones et leurs appareils photo intégrés et connectés à différentes applications.

\subsection{Une « textualité mobile»}

Il y a deux grands types de photographies de texte qui circulent sur les réseaux sociaux et les messageries: la photographie manuelle d'une page, d'un livre, d'un document quelconque, à l'aide d'un appareil photo (voir ci-dessous les images 10 à 16), et la capture d'écran ou screenshot de texte à l'aide des commandes d'un ordinateur ou de logiciels et 
applications spécifiquement dédiés à la capture d'écran permettant aussi le surlignement, l'annotation, le classement, etc.

Dans le premier cas on photographie le texte comme objet matériel, dans le second on produit un extrait de texte d'écran à écran sans trace de matérialité ; dans les deux cas le texte est iconisé. J'ai traité ces deux types de technographisme dans L'analyse du discours numérique (entrée Technographisme), et montré qu'ils étaient dotés des traits technodiscursifs suivants : ils constituent des technodiscours rapportés et des pratiques de citation numériquement outillées (traits énonciatifs), il accomplissent un embarquement du contexte (trait pragmatico-discursif) et une numérisation native profane du texte (trait morpho-typographique et bibliomatériel). Ils assument plusieurs fonctions dont le contournement de la limitation de format (dans les cas de limite de caractères comme sur Twitter) ou de la contrainte de l'abonnement (un screenshot d'article de presse réservé aux abonnés est une forme de hacking), la transmission d'informations à distance (une référence, un numéro de page, un article de loi).

Les photographies ou les screenshots de texte à partir d'un smartphone constituent selon Laurence Allard une "textualité mobile ", caractérisée par une «indifférenciation entre iconique et scriptural » (Allard 2014b: 148). L'enquête ethnographique qu'elle a menée auprès d'usagers de smartphone souligne des pratiques courantes de photographies de texte: «De nombreux récits d'usagers font part de leurs pratiques de photographies de billets de train ou d'avions, de passeports ou d'étiquettes de bagages, d'annonces immobilières, de listes de courses, etc. » (Allard 2014b : 148). Elle note par ailleurs, comme André Gunthert, la fonction conversationnelle de l'image: "Converser par l'intermédiaire d'une image mobile pour dire quelque chose de soi et du monde est l'un des procédés discursifs les plus courants sur les sites de réseaux sociaux [...] " (Allard 2014b : 151). La notion de textualité mobile, assortie de celle de «mobtexte» que la chercheuse mobilise également (Allard 2015) me semble une contribution intéressante à la réflexion sur le faire texte contemporain connecté : outre «l'indifférenciation » imagetexte, que je nomme pour ma part composition donnant lieu à une iconisation du texte, le texte mobile est "perçu comme un texte activable dans une logique typique du numérique de "manipulabilité" " (Allard 2014b : 149). La manipulabilité du texte-image, technographisme qui se fait textographisme, est en effet un trait majeur d'une textualité numérique pilotée par l'image : le technographisme est principalement destiné à circuler, posté ou multiposté 9 sur les réseaux sociaux. L'iconisation du texte est une circulation d'images.

Je choisis d'examiner ici un type de photographie de texte qui ne se contente pas seulement d'iconiser le texte mais qui est aussi marqué par une énonciation visuelle en première personne : la photographie déictique de texte comporte un élément corporel ou subjectif du.de la photographe, qui l'installe comme énonciateur.trice visuel.le, le plus souvent une main. Cette configuration énonciative visuelle me semble interroger à la fois la théorie du texte et celle de l'énonciation dans leurs versions encore prédominante en analyse des textes et des discours.

\subsection{L'iconisation de la deixis}

J'examine ici la photographie déictique en général avant de revenir précisément sur la photographie de texte. 
J'utilise la notion de deixis au sens littéral qu'elle a en linguistique énonciative : un énoncé déictique est un énoncé autoréférentiel qui porte dans sa forme matérielle les marques du je, et/ou de l'ici et/ou du maintenant de l'énonciateur.trice. De même, les photographies déictiques portent iconiquement les marques du je par l'intermédiaire du fragment de corps du.de la photographe. Ce sont toujours des autophotographies, c'est-àdire que le fragment de corps est photographié par le sujet et non par un tiers; ce ne sont pas des photos en troisième personne, mais bien en première personne. Il peut arriver que le fragment de corps soit absent, mais qu'un cadrage fortement subjectif, impliquant une position particulière du corps du.de la photographe (position agenouillée, couchée, torsion, etc.,). Les plus fréquentes et stéréotypées en ligne sont sans doute les selfies, la deixis étant portée par l'exhibition du mouvement ou de la trace de mouvement du bras portant l'appareil (Gunthert 2015). La photographie déictique constituant un genre photographique à part entière, d'autres modèles se trouvent en masse sur les réseaux, comme la photographies de pieds (voir image 6), la photographie de transport (fenêtre de train ou de métro, aile d'avion, comme dans l'image 8 dont le commentaire pointe d'ailleurs la stéréotypie), de café (une table, une tasse ou un verre, un livre ou un carnet, une main, comme dans l'image 9) ou de texte, qui fait l'objet du développement suivant ${ }^{10}$.

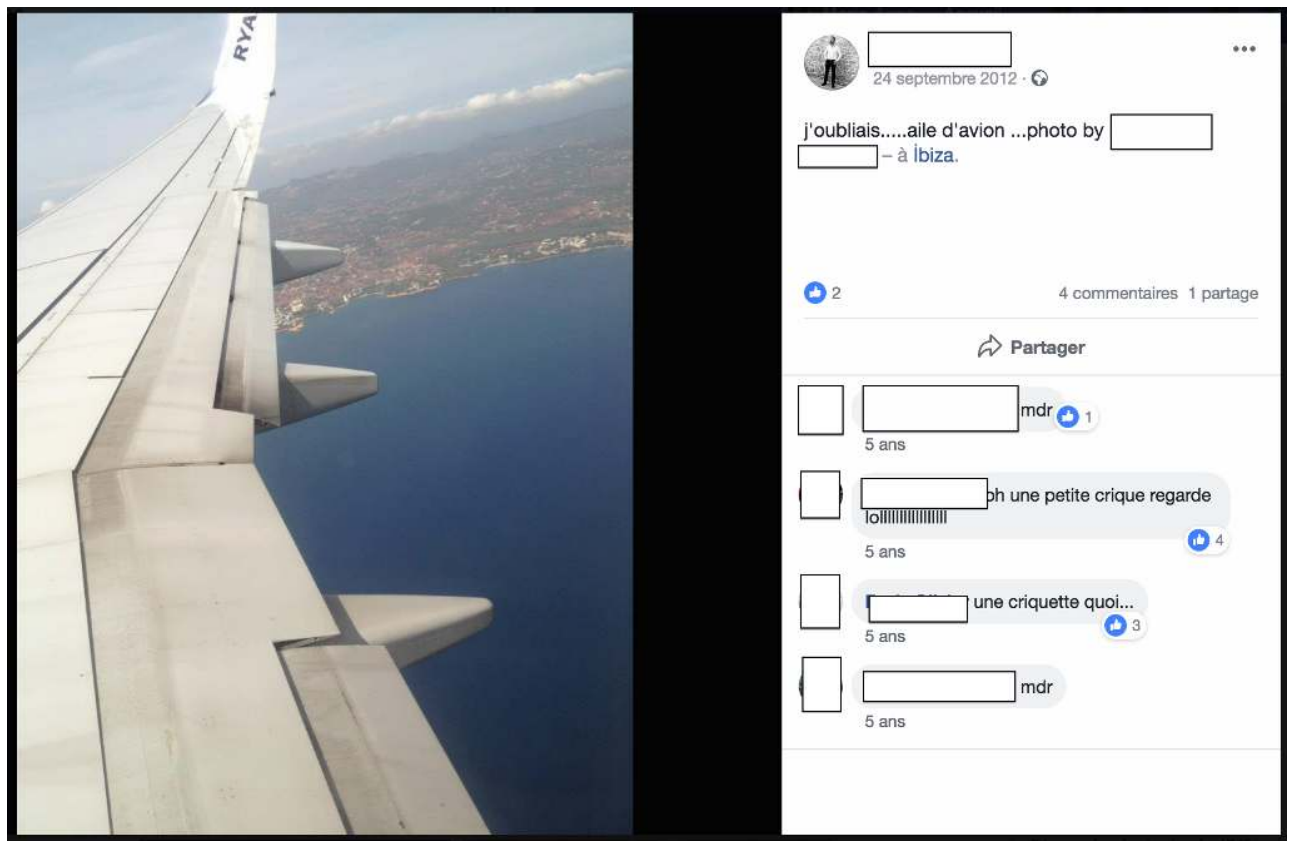

Image 8. Photographie déictique de transport : l'aile d'avion (Facebook) 


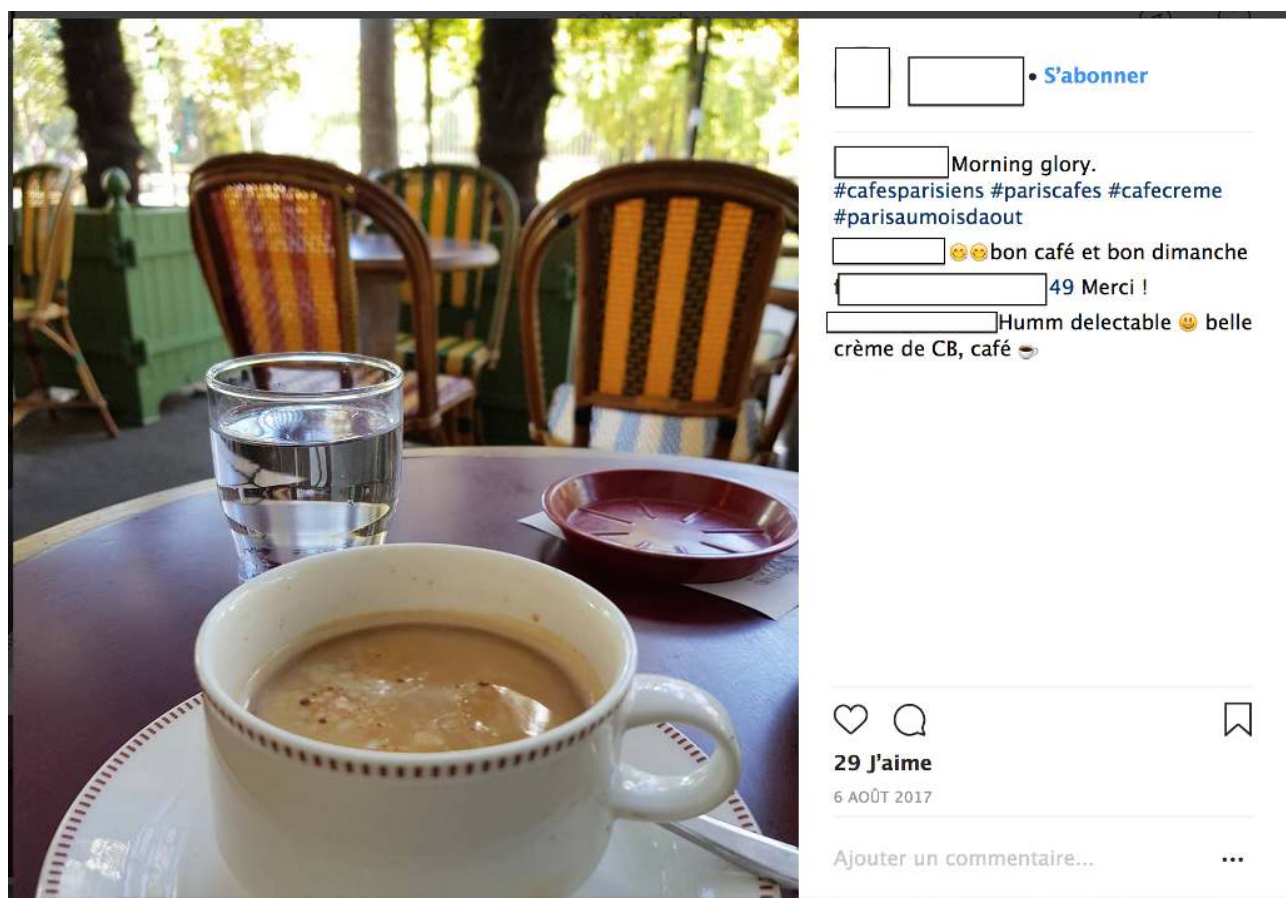

Image 9. Photographie déictique de café (Instagram)

On peut alors dire que la deixis elle-même est iconisée, retrouvant en cela d'ailleurs son sens étymologique (montrer de la main) : de marque langagière pronominale, on passe à une marque iconique corporelle, qui joue exactement le même rôle.

\subsection{Morphologie énonciative de la photographie déictique de texte}

Les photographies déictiques de texte sont de deux ordres selon que la deixis est explicite (présence d'une partie du corps) ou implicite (portée par l'angle de prise de vue marquant fortement la subjectivité du cliché).

\subsubsection{La photographie de texte avec deixis explicite}

33 Je redis que ce type d'exemple est étroitement dépendant du profil socioculturel de mes contacts sur Facebook, Instagram et Twitter, et que je présente plutôt des pratiques lettrées d'universitaires, de journalistes ou d'écrivain.e.s. Mais la photographie de texte ne se limite pas aux usages cultivés, comme le montre Laurence Allard dans son enquête sur les usages mobiles: plusieurs enquêté.e.s l'informent de photographies de textes ordinaires et utilitaires (Allard 2014b). La photographie de texte est donc une pratique générale, qui, comme la lecture ou l'écriture, possède ses versions plus ou moins marquées par les appartenances sociales, culturelles et générationnelles. Les trois exemples que je présente ici concernent trois sous-pratiques distinctes: la lecture, l'écriture manuelle et la numérisation de texte. 


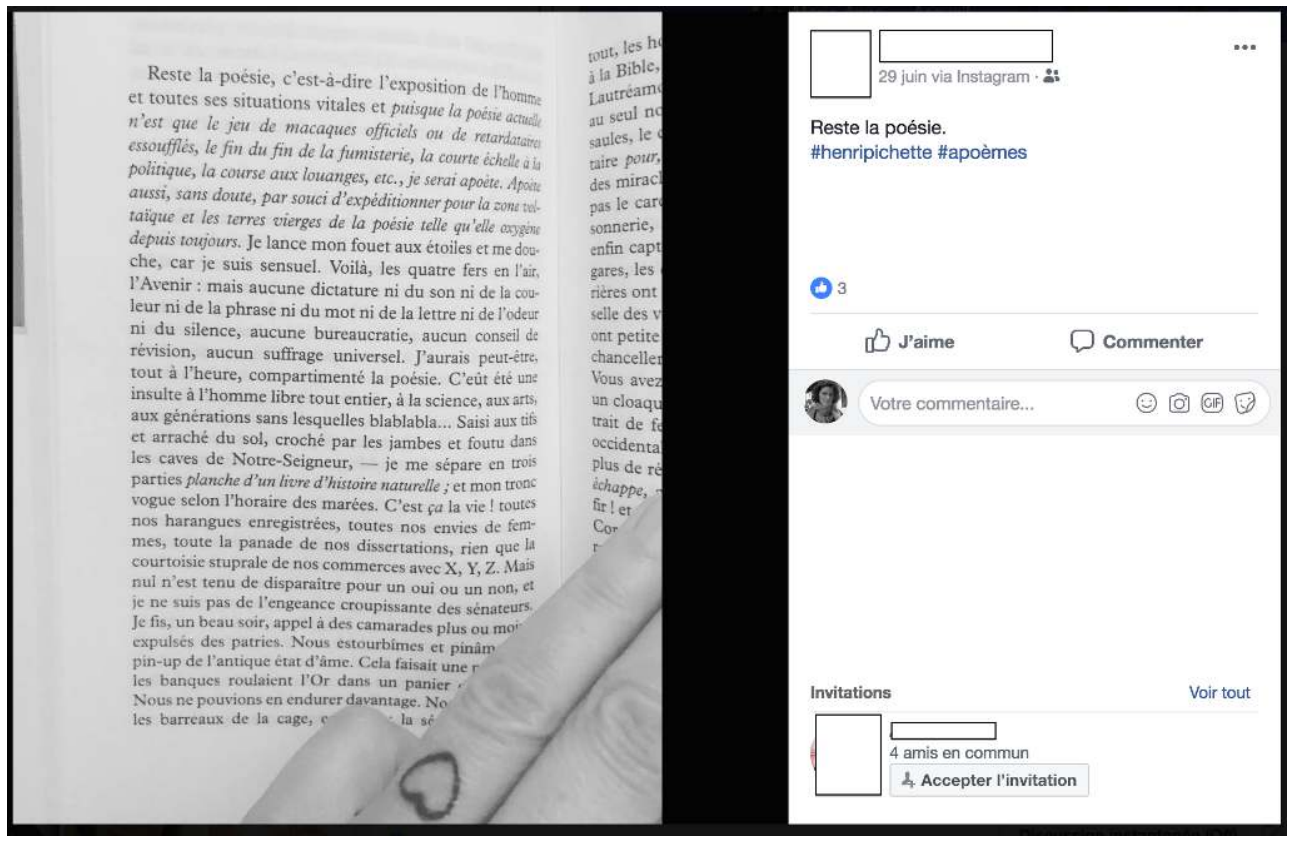

Image 10. Photographie de texte, deixis explicite, lecture de livre (Facebook, juin 2018)

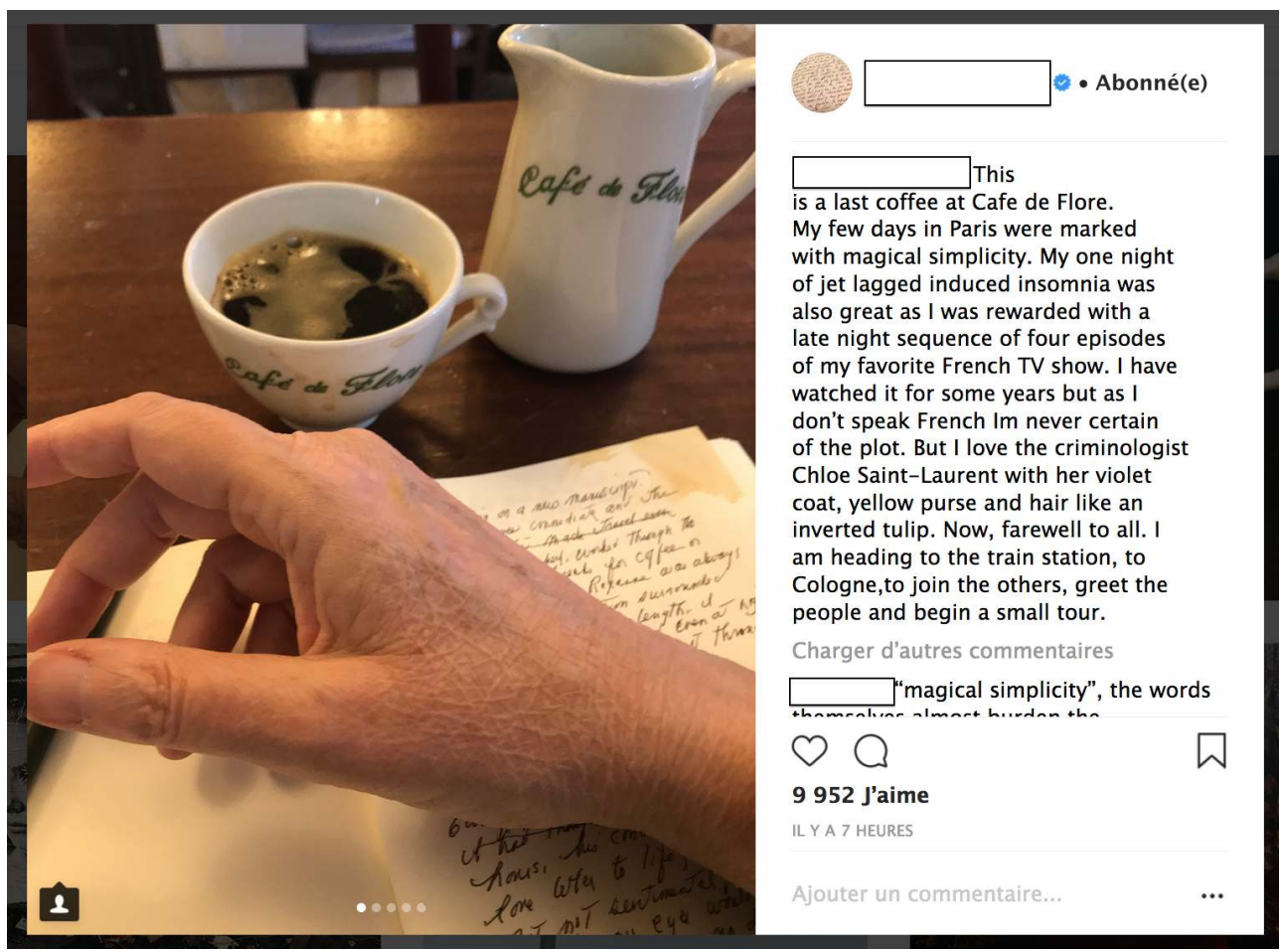

Image 11. Photographie de texte, deixis explicite, écriture (Instagram, juillet 2018) 


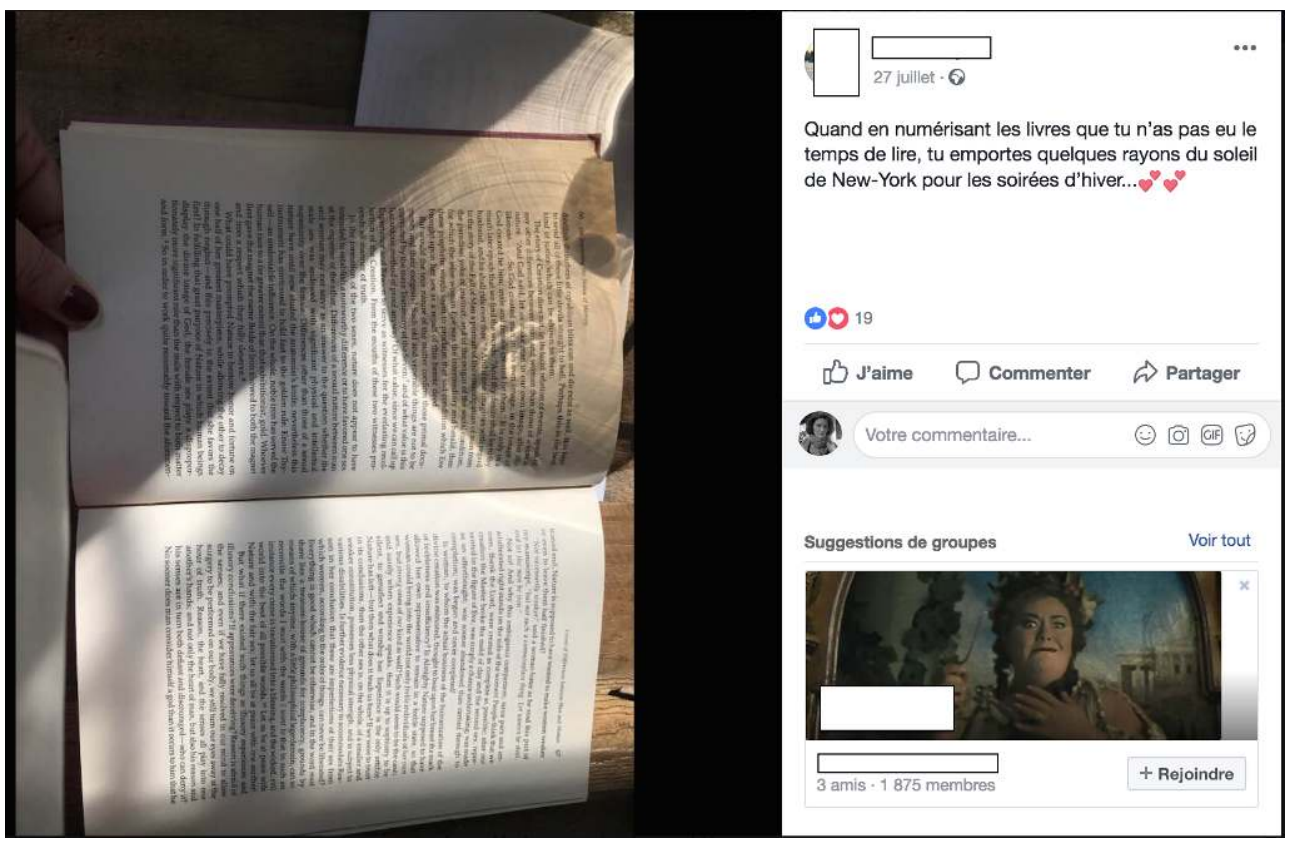

Image 12. Photographie de texte, deixis explicite, numérisation de livre (Facebook, juillet 2018)

Dans ces trois exemples apparaissent explicitement la marque iconique corporelle, doigt et main, qui fonctionne comme marqueur déictique de présence. Les textes iconisés, pages de livre ou de carnet, ont des statuts différents, proposé à la lecture pour le premier, élément de micro-narration ou de mise en scène pour les deux suivants ${ }^{11}$. Dans les trois cas, on a une double iconisation, de l'énoncé et de l'énonciation.

\subsubsection{La photographie de texte avec deixis implicite}

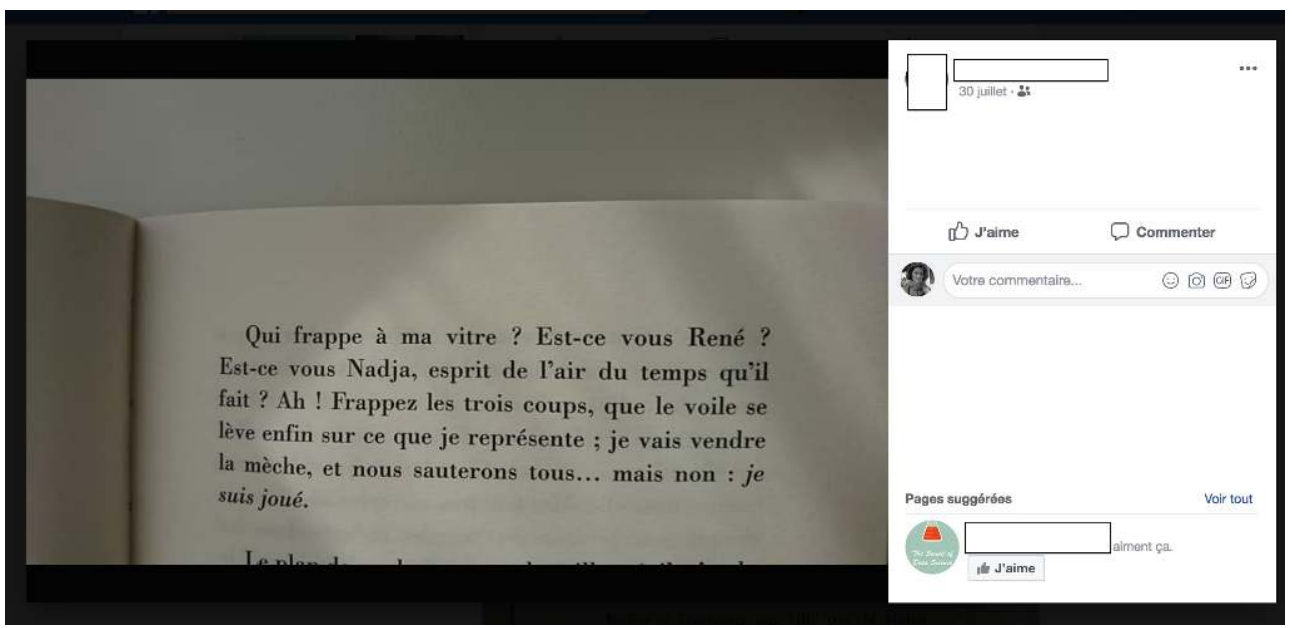

Image 13. Photographie de texte, deixis implicite, lecture (Facebook, juillet 2018) 


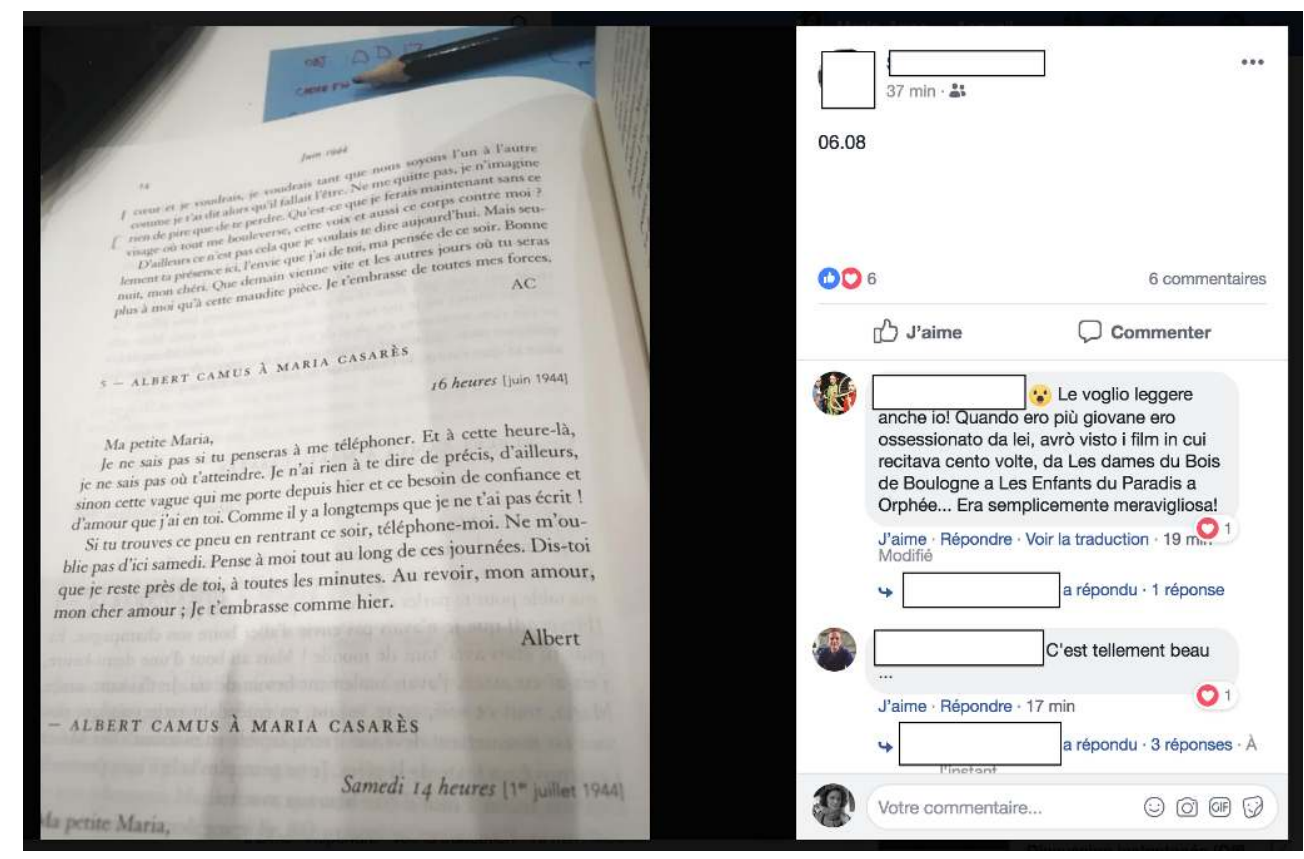

Image 14. Photographie de texte, deixis implicite, lecture (Facebook, août 2018)

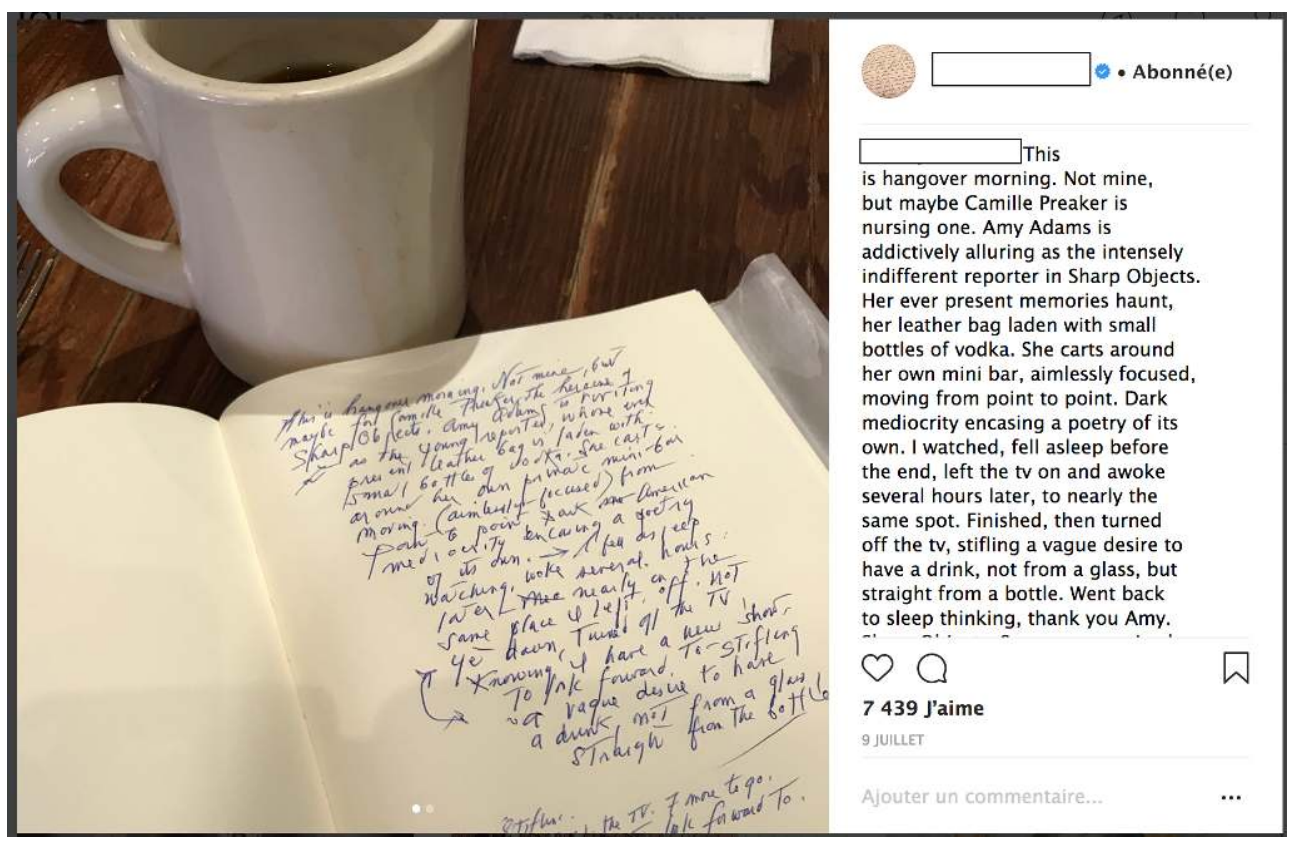

Image 15. Photographie de texte, deixis implicite, écriture (Instagram, juillet 2018)

Dans ces trois exemples, les marques iconiques corporelles sont absentes mais non les marqueurs déictiques de présence. Dans l'image 13, le cadrage en gros plan, le creux du livre ouvert et tenu hors champ et les ombres sur la page constituent des indices de présence du.de la photographe énonciateur.trice. Dans l'image 14, ce sont l'angle de prise, les ombres et en particulier celle du smartphone qui apparait en bas de l'image. Dans l'image 15 , qui correspond à un modèle très fréquent sur les réseaux, c'est le cadrage en gros plan et en légère plongée qui assurent la présence déictique. On peut donc parler de photographie déictique de texte même si les indices corporels explicites ne figurent pas sur l'image. 


\subsubsection{Technographisme et deixis}

à examiner le rapport que les images déictiques entretiennent ave produits dans les posts, qui figurent dans quatre des six exemples ci-dessus. Je ne vais pas entrer dans le détail de cette question traitée par ailleurs à propos du gif animé (Paveau 2018). Je voudrais simplement souligner ici que les énoncés dans les posts et les photographies déictiques ont un rapport composite. En effet, les énoncés ne sont pas des légendes qui accompagneraient a posteriori les images, et inversement les images ne sont pas des illustrations des textes; l'ensemble forme une seule et même unité technographique complexe, la double iconisation de l'image étant assortie d'énoncés qui leurs sont isoréférentiels, c'est-à-dire doublant de différentes manières le contenu de l'image. Dans l'image 10, l'incipit de la page est simplement répété ; les commentaires des images 11 et 15 , commençant par «this is » et se continuant en minirécits, prédiquent le contenu des images de manière déictique; l'énoncé de l'image 12, commençant par le quand tu stéréotypé $d u$ « dialecte » des réseaux, présente également de manière déictique le contenu de l'image (la construction quand tu équivaut à peu près à un voici). Dans les images 11, 12 et 15, la deixis est donc doublement présente, dans le contenu de la photographie et dans le rapport entre l'énoncé verbal du post et la photographie. La deixis est donc un élément doublement structurant de la photographie de texte dans cette configuration.

\section{Conclusion}

37 J'ai décrit dans ce travail un phénomène qui me semble caractéristique des discours numériques natifs, mais qui se place dans une évolution sémiotique plus large: l'énonciation matérielle visuelle. Elle s'inscrit dans une forme qui assemble image et texte de manière composite, le technographisme, dans une représentation du texte par la photographie et la capture d'écran qui assure sa conversion iconique, et dans un mode d'énonciation qui mobilisation une deixis corporelle, elle-même iconisée. L'énonciation visuelle repose donc sur une iconisation des discours, sur les trois plans de leur forme, de leur représentation et de leur énonciation.

Ce phénomène a des implications fortes pour la linguistique, qui ne peut plus rester dans les périmètres du logos, mais doit prendre en compte les évolutions des formes d'expression telles qu'elles se produisent dans les univers numériques natifs. Sur le plan linguistique, la conversion numérique décrite par Milad Doueihi est une iconisation et il est patent que les écosystèmes numériques natifs favorisent le pictorial turn du langage articulé. Pour rendre compte de ce phénomène, la linguistique doit sans doute élargir son empan disciplinaire en intégrant des observations et propositions théoriques d'autres disciplines, comme les sciences de l'information et de la communication, les études visuelles ou la psychologie cognitive. Elle pourrait également envisager une approche écologique des phénomènes langagiers et discursifs, qui tienne compte de l'ensemble de leurs environnements et non plus de leurs seules formes saisies à travers le prisme du système de la langue.

Corela, HS-28 | 2019 


\section{BIBLIOGRAPHIE}

N.B. les liens ont été vérifiés le 10.08.2018

Adam, Jean-Michel (dir.), Faire texte. Unité(s) et (dis)continuité. Besançon : PUFC.

Allard Laurence, 2014a, « Le selfie, un genre en soi. Ou pourquoi, il ne faut pas prendre les selfies pour des profil pictures », Mobactu [blog] : http://www.mobactu.fr/?p=904

Allard Laurence 2014b, « Express Yourself 3.0! Le mobile comme technologie pour soi et quelques autres entre double agir communicationnel et continuum disjonctif soma-technologique », dans Allard Laurence, Creton Laurent, Odin, Roger (dir.) Téléphone mobile et création, Paris, Armand Colin, 139-162.

Bouchardon Serge et al., 2011, « Explorer les possibles de l'écriture multimédia », Les Enjeux de l'information et de la communication 12/2:11-24.

Cabourg Céline, Manenti Boris, 2017, Portables : la face cachée des ados, Paris, Flammarion.

Casilli Antonio, 2012, « Être présent en ligne : culture et structure des réseaux sociaux d'Internet ", Idées économiques et sociales, 169, p. 16-29.

Clark Alan, Chalmers David, 1998, « The extended mind », Analysis 58 (1) : 10-23.

Dervin Fred, Paveau Marie-Anne, 2012 (dir.) : Quelle place pour les objets dans les sciences du langage et sciences de la communication? Synergies Pays Riverains de la Baltique 9, https:// gerflint.fr/Base/Baltique9/baltique9.html

Doueihi Milad 2008, La grande conversion numérique, trad. de l'anglais par P. Chemla, Paris, Seuil.

Gibson James J., 1977, « The Theory of Affordances » in R. Shaw and J. Brandsford (eds), Perceiving, Acting, and Knowing : Toward an Ecological Psychology, 62-82.

Gunthert André, 2013, « Il neige sur Facebook », L'Atelier des icônes, Carnet de recherches d'André Gunthert (archive), http://histoirevisuelle.fr/cv/icones/2606

Gunthert André, 2014, «L'image conversationnelle », Études photographiques, 31, http:// etudesphotographiques.revues.org/3387

Gunthert André, 2015, « La consécration du selfie », Études photographiques 32, http:// etudesphotographiques.revues.org/3529

Hutchins Edwin, 1995, Cognition in the Wild, Bradford Books-MIT Press, Cambridge MA.

Jeanneret Yves, Souchier Emmanuël, 2005, «L'énonciation éditoriale dans les écrits d'écran », Communication et langages 145 : 3-15.

Ibrahim Yasmin, 2015, « Food Porn and the Invitation to Gaze : Ephemeral Consumption and the Digital Spectacle », International Journal of E-Politics $6 / 3: 1-12$.

Latour Bruno, 1991, Nous n'avons jamais été modernes. Essai d'anthropologie symétrique, Paris, La découverte.

Mitchell William J. Thomas, 2009 [1986], Iconology : Image, Text, Ideology, Chicago, The University of Chicago Press, 1986. Iconologie. Image, texte, idéologie, trad. de l'anglais (USA) par Maxime Boidy et Stéphane Roth, Paris, Les Prairies ordinaires. 
Mitchell William J. Thomas, 1994, Picture Theory: Essays on Verbal and Visual Representation, Chicago, The University of Chicago Press.

Nachtergael Magali, 2017, « Le devenir-image de la littérature : peut-on parler de "néolittérature" "? dans Mougin Pascal dir., La tentation littéraire de l'art contemporain, Presses du réel : 291-304.

Nerlich Michael, 1990, «Qu'est-ce qu'un iconotexte ? Réflexions sur le rapport texte-image photographique dans La Femme se découvre d'Evelyne Sinnassamy » dans Alain Montandon (éd.), Iconotextes, Paris, Ophrys : 255-302.

Norman Don, 1993, « Les artefacts cognitifs » dans Conein B., Dodier N., Thevenot L., 1993, Les objets dans l'action. De la maison au laboratoire, série « Raisons pratiques ", $n^{\circ} 4$, Paris, éd. de l'EHESS : 15-34.

Paveau Marie-Anne, 2012, "Ce que disent les objets. Sens, affordance, cognition », Synergies Pays Riverains de la Baltique 9, p. 53-65, http://ressources-cla.univ-fcomte.fr/gerflint/Baltique9/ baltique9.html

Paveau Marie-Anne, 2013, « Technodiscursivités natives sur Twitter. Une écologie du discours numérique », Epistémè 9 : 139-176.

Paveau Marie-Anne, 2015a, « Ce qui s'écrit dans les univers numériques. Matières technolangagières et formes technodiscursives ", Itinéraires ltc : http:// itineraires.revues.org/2313

Paveau Marie-Anne, 2015b, « En naviguant en écrivant. Réflexions sur les textualités numériques », dans Adam J.-M. (dir.), Faire texte. Unité(s) et (dis)continuité, Besançon, PUFC : 339-355.

Paveau Marie-Anne, 2017a, L'analyse du discours numérique. Dictionnaire des formes et des pratiques, Paris, Hermann.

Paveau Marie-Anne 2017b, « Du microscope à la caméra subjective. Les observables natifs de l'internet ", Le discours et la langue 9.2. : 160-172.

Paveau Marie-Anne, 2018, «L'image du corps comme énonciation. Le cas des gifs corporels sur les réseaux sociaux », communication au $3^{\mathrm{e}}$ colloque IMPEC, Lyon, 4-6.07.2018.

Pétonnet Colette, 1982, « L'Observation flottante. L'exemple d'un cimetière parisien », L'Homme 22-4, Etudes d'anthropologie urbaine : 37-47.

Souchier, Emmanuël, 1996, « L'écrit d'écran, pratiques d'écriture \& informatique ». Communication et langages $107: 105-119$.

Souchier, Emmanuël, 1998, «L'image du texte. Pour une théorie de l'énonciation éditoriale », Les Cahiers de médiologie $6: 137-145$.

\section{NOTES}

1. Je rappelle que web et internet ne sont pas synonymes et que le web n'est qu'un des services d'internet comme la messagerie électronique, l'échange de fichiers ou les forums de discussion. Mon terrain est ici principalement le web 2.0 ou web social ou web conversationnel, saisi à partir des réseaux sociaux numériques les plus pratiqués, Twitter, Facebook et Instagram. 
2. La notion de symétrie est tirés des travaux de Bruno Latour, en particulier dans Nous n'avons jamais été modernes. Essai d'anthropologie symétrique, où il définit le rapport entre nature et culture comme une prise en compte égale des deux ordres et non plus leur opposition (Latour 1991).

3. La notion de médiation est encore très présente chez les linguistes qui travaillent sur le discours numérique ou électronique, connecté ou pas, via les pratiques de la CMO (Communication médiée par ordinateur) née dans les années 1990 à la suite de la CMC (Computer mediated communication) anglophone.

4. Image postée le 18.06.2013 sur le site Pod Academy : http://podacademy.org/2013/humanitiesand-social-sciences/i-need-feminism-because/

5. La redocumentation est une pratique numérique native réflexive qui consiste à archiver, classer et mettre en mémoire les contenus du web social, par différents moyens manuels ou logiciels. Le web social est un lieu de collections, d'anthologies, et de listes en tous genres qui témoignent d'un auto-ordonnancement continu des contenus publiés.

6. La notion d'observation flottante est proposée par l'anthropologue Colette Pétonnet en 1982 dans une étude sur un cimetière parisien, dans le contexte du début des études urbaines en France. Elle « consiste à rester en toutes circonstances vacant et disponible, à ne pas mobiliser l'attention sur un objet précis, mais à la laisser "flotter" afin que les informations la pénètrent sans filtre, sans a priori, jusqu'à ce que des points de repère, des convergences, apparaissent et que l'on parvienne alors à découvrir des règes sous-jacentes » (Pétonnet 1982 : 39).

7. L'homophilie désigne en sociologie la tendance à s'associer avec des personnes qui nous ressemblent. Sans être prépondérante sur les réseaux sociaux numériques, elle détermine cependant un certains nombres de liens (sur cette question voir Casilli 2012.

8. L'écrilecture désigne la simultanéité des actes de lecture et d'écriture dans les textes en ligne, notamment du fait des liens hypertexte qui font du.de la lecteur.trice un.e scripteur.e.

9. Le terme multiposté se réfère au phénomène de la publication multisite, qui consiste à poser simultanément dans plusieurs espaces la même production à l'aide d'outils dédiés.

10. Je n'intègre pas la photographie de nourriture, pourtant extrêmement fréquente, à la photographie déictique pour deux raisons: d'abord elle s'inscrit dans une catégorie installée depuis une dizaine d'années dans les univers numériques, le foodporn, dont l'objectif prioritaire est de représenter la nourriture et non le soi ; ensuite, et conséquemment, les photographies de foodporn contiennent rarement des éléments corporels du.de la photographe, constituant majoritairement des photographies en troisième personne. Dans le foodporn en effet, les photographies sont centrées sur l'objet et ses effets de désirabilité et sur non le.a photographe ; elles sont la plupart du temps centrées en gros plan, sans élément contextuel, autrement dit, elles sont centrées sur l'énoncé et non sur l'énonciation, même si la photographie de nourriture, devenue un véritable objet culturel sur le web, constitue aussi une image conversationnelle (Ibrahim 2015).

11. Pour les traits et fonctions linguistiques des textes iconisés, voir Paveau 2017, entrée Technographisme.

\section{RÉSUMÉS}

Dans le cadre des visual studies, Mitchell (1994) a montré que l'image organise de plus en plus notre perception sémiotique, en réarticulant la production du sens dans une perspective 
iconique. L'hypothèse d'un « devenir-image » (Nachtergael 2017) du texte, du discours et de l'interaction n'est donc pas nouvelle mais semble confirmée par la communication numérique, où l'image joue un rôle important, réorganisant les contenus de sens et prenant parfois le pas sur l'expression verbale au sein du technographisme, défini comme une production multimédiatique numérique native articulant image, texte et éventuellement son (Paveau 2017). Dans cet article, je propose de mettre en œuvre cette hypothèse à partir de l'exemple de la photographie de texte imprimé ou sur écran, qui est de plus en plus intégrée dans les discours numériques natifs, en particulier sur les réseaux sociaux comme Twitter ou Facebook. Je montrerai qu'elle relève d'une iconisation du texte, témoignant d'une conversion numérique de la scripturalité et posant la question d'un pilotage contemporain du sens par l'image.

In the context of visual studies, Mitchell (1994) showed that the image increasingly organizes our semiotic perception, by re-correlating the production of meaning in an iconic perspective. The hypothesis of an "image-becoming" (Nachtergael 2017) of text, discourse and interaction is therefore not new but seems to be confirmed by digital communication, where image plays an important role, reorganizing meaningful content and sometimes taking precedence over verbal expression within technographism, defined as a native digital multimedia production articulating image, text and possibly sound (Paveau 2017). In this article, I propose to implement this hypothesis using the example of printed or on-screen text photography, which is increasingly integrated into native digital speech, especially on social networks such as Twitter or Facebook. I will show that it is an iconization of the text, testifying to a digital conversion of scripturality and raising the question of a contemporary piloting of meaning through the image.

\section{INDEX}

Mots-clés : discours numérique, iconisation du texte, pictorial turn, scripturalité, technographisme

Keywords : digital discourse, text iconization, pictorial turn, scripturality, technographism

\section{AUTEUR}

\section{MARIE-ANNE PAVEAU}

Université Paris 13 